\title{
High resolution simulations of energy absorption in dynamically loaded cellular structures
}

\author{
R. E. Winter ${ }^{1}$ - M. Cotton ${ }^{1}$ - E. J. Harris ${ }^{1}$ - D. E. Eakins ${ }^{2}$ - G. McShane ${ }^{3}$
}

Received: 1 October 2015 / Revised: 2 February 2016 / Accepted: 9 March 2016 / Published online: 19 April 2016

(C) The Author(s) 2016. This article is published with open access at Springerlink.com

\begin{abstract}
Cellular materials have potential application as absorbers of energy generated by high velocity impact. CTH, a Sandia National Laboratories Code which allows very severe strains to be simulated, has been used to perform very high resolution simulations showing the dynamic crushing of a series of two-dimensional, stainless steel metal structures with varying architectures. The structures are positioned to provide a cushion between a solid stainless steel flyer plate with velocities ranging from 300 to $900 \mathrm{~m} / \mathrm{s}$, and an initially stationary stainless steel target. Each of the alternative architectures under consideration was formed by an array of identical cells each of which had a constant volume and a constant density. The resolution of the simulations was maximised by choosing a configuration in which onedimensional conditions persisted for the full period over which the specimen densified, a condition which is most readily met by impacting high density specimens at high velocity. It was found that the total plastic flow and, therefore, the irreversible energy dissipated in the fully densified energy absorbing cell, increase (a) as the structure becomes more rodlike and less platelike and (b) as the impact velocity increases. Sequential CTH images of the deformation processes show that the flow of the cell material may be broadly divided into macroscopic flow perpendicular to the compression direction and jetting-type processes (microki-
\end{abstract}

Communicated by P. Hazell.

$\triangle$ R. E. Winter

rwinter1@imperial.ac.uk

1 AWE, Aldermaston, Reading, Berkshire RG7 4PR, UK

2 Institute of Shock Physics, Imperial College London, London, UK

3 Department of Engineering, University of Cambridge, Cambridge, UK netic flow) which tend to predominate in rod and rodlike configurations and also tend to play an increasing role at increased strain rates. A very simple analysis of a configuration in which a solid flyer impacts a solid target provides a baseline against which to compare and explain features seen in the simulations. The work provides a basis for the development of energy absorbing structures for application in the $200-1000 \mathrm{~m} / \mathrm{s}$ impact regime.

Keywords Cellular structures - Additive manufacture . Impact $\cdot$ Shock

\section{Introduction}

A common requirement in the design of many structures and vehicles is the ability to ameliorate the effects of projectile impact or collisions. In particular, scenarios in which a high velocity projectile impacts a target may require stresses to be minimised in either the projectile or the target or, sometimes, both. This protection is often accomplished by incorporating a "cushion" of porous or cellular materials between the projectile and the target. This energy absorbing component can act to dissipate the excess energy via irreversible dynamic deformation processes.

Porous or cellular materials have demonstrated superior energy absorption under shock or impact loading when compared to monolithic materials [1,2], while also offering the benefit of improved strength to weight ratios. A variety of cellular structures have been investigated with the aim of improving understanding of compressive response and thus maximising energy absorption over a range of representative impact conditions. These include metal [3-5] and polymeric [6-9] foams, composites [10-12] and honeycombs [13-15]. One notable feature of this class of materials is the enhance- 
ment of crushing strength observed under dynamic loading due to inertial effects, as seen by Reid and Peng [16] in wood, Tan et al. [17] in aluminium foams and Xue and Hutchinson [18] and Wu and Jiang [19] in metallic honeycombs. This phenomenon has also been the subject of a numerical study by Liu et al. [20], the conclusions of which identify a critical velocity above which inertial effects become significant.

Recent investigations have expanded the range of structures to include lattice truss structures such as octet [21], Kagomé [22] or pyramidal [23] designs. For a review of the various core topologies and manufacturing methods employed in the creation of periodic cellular structures the reader is referred to Wadley [24] and Lefebvre et al. [25]. Many of these studies fielded the cellular material in a sandwich panel configuration whereby the deflection of the face sheets can be used as a metric for measuring energy absorption.

Advances in manufacturing technology, in particular the flexibility of design afforded by additive manufacturing, have led to a significant increase in the range of cellular geometries available, for example the gyroid structure as described by Yan et al. [26], or microlattice designs tested by Mines et al. [27]. Such complex structures can be realised due to the ability of additive processes to build components as an aggregate of thin layers of material, using a three-dimensional (3D) Computer Aided Design (CAD) model as a reference. As a result of these developments, there is a growing requirement to compare the relative merits of different structures and identify the most appropriate for a given loading scenario.

As described above, previous research on the response of cellular structures, both additively and conventionally manufactured, has improved our understanding of energy absorbing processes in this important class of materials and has led to refinement of cellular material design. In particular in many of the past studies, computer simulation has been used both to highlight the important deformation phenomena and to optimise material design. Clearly, an important prerequisite of such simulations should be to determine how well the chosen computational technique matches experiments performed in the appropriate loading regime. It is especially important to determine the validity of the computer code during the initial phase of the compression, in which the plastic wave generated in the lattice by the impact propagates through the full thickness of the sample. This is because, if the code cannot model the early phase of the deformation, it is very unlikely that it will model the later phases correctly. However in many previous studies the response of the system is affected by stress (usually release) waves, propagating from the lateral outer boundaries of the sample before the initial plastic wave traverses the thickness of the specimen. For example, consider a disc-shaped cellular metal sample of diameter $200 \mathrm{~mm}$ and thickness $40 \mathrm{~mm}$ impacted

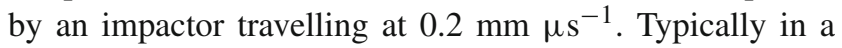

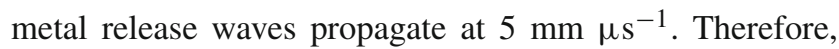
following impact, release waves will travel the $100 \mathrm{~mm}$ from the outer boundary to the centre of the system in $20 \mu \mathrm{s}$. During this $20 \mu \mathrm{s}$ period the flyer will travel $4 \mathrm{~mm}$ or about $10 \%$ of the thickness of the specimen. It follows, therefore, that for most of the period of compression, the response of the sample, including the region near the centre of the sample, depends on the position of the outer perimeter. Therefore, to allow meaningful comparison between experiment and simulation the whole sample must be included in the model. Further if the detailed response of the individual struts or other features of the cells is needed, then, in general, a threedimensional (3D) code must be used. In summary, in the two or three-dimensional configurations employed in much of the previous work on this topic, the need to model the whole experiment plus the requirement to model the structure of the individual cells in 3D would require unrealistically high computational resources.

\section{Advantages of one-dimensional (1D) geometry}

Significant computing resources can be saved in the simulation of cellular structures if a "pseudo 1D" modelling scheme similar to that employed in simulations by Borg and Vogler [28] is adopted. Consider a cylindrical sample made up of a regular cellular structure in which the diameter of the sample is much greater than its thickness. It can be shown that, following impact, the time to reach full densification of the whole specimen is less than the time for release waves to reach the axis of the sample if the diameter of the specimen, the density of the sample and the impact velocity are high and the thickness of the specimen is low. Under such conditions, the compression of the cells at the centre of the disc may, on a macroscopic or continuum scale, be considered 1D. At the scale of individual cells such a system is, of course, not 1D but 3D. However, cells near the centre of the disc will experience the same stress field as each other and therefore every cell will respond in the same way. In this situation, modelling a single cell or column of cells provides the response of the whole of the region over which 1D conditions apply. Note that since, in general, the deformation of an individual cell is three dimensional, rather than one dimensional, the configuration should, perhaps, be more correctly termed "Pseudo 1D". However, for convenience we will refer to this modelling concept as "1D" during the remainder of this paper. Clearly significant computing resources are saved by confining modelling to a single cell or small group of cells.

In an earlier paper, we described a study in which lattices consisting of an array of intersecting stainless steel rods were manufactured using an additive manufacturing technique known as Selective Laser Melting (SLM) [29]. The structure consisted of intersecting $316 \mathrm{~L}$ stainless steel rods. The sam- 
ples, which were accelerated using a $100 \mathrm{~mm}$ gas gun, were discs of diameter $\sim 100 \mathrm{~mm}$ and thickness $6 \mathrm{~mm}$. The cells which made up the lattice measured $1 \mathrm{~mm} \times 1 \mathrm{~mm} \times 1 \mathrm{~mm}$ and the average density of the lattice was $64 \%$ of solid. Impact velocities ranged from 0.3 to $0.7 \mathrm{~mm} \mathrm{\mu s}^{-1}$. A rough estimate of the time for which 1D conditions persist in this geometry is as follows. The time for elastic release waves from the perimeter to reach the centre of the system was $\sim 10 \mu \mathrm{s}$. At an impact velocity of $0.5 \mathrm{~mm} \mathrm{\mu s}^{-1}$ the time for full densification of the lattice was of the order of $6 \mu \mathrm{s}$. Therefore a useful amount of compression was achieved before the effect of release waves from the outer of the specimen was felt at the centre of the system. Note that the densities of the sample, and the impact velocity regime in our work are somewhat higher than in many previous studies of the dynamic response of cellular materials. In this regime the behaviour of the material tends to be dominated by shock formation and propagation and as such relates to severe blast and impact scenarios.

The experiments were simulated using the Sandia National Laboratories Code CTH. CTH is a multi-dimensional, multimaterial Eulerian-based hydro-code developed by Sandia National Laboratories [30]. The Eulerian meshing scheme enables it to cope with the simulation of large deformations and material distortions. The incorporation of the laws of conservation of mass, momentum and energy, together with a suite of material models which allow description of most states of matter normally encountered in shock physics, make the code appropriate for calculating the response of materials loaded under shock and high strain rate conditions. In effect the code is optimised for simulating situations, encountered at high impact energies, in which inertial (or material acceleration) effects dominate. The effects of material strength on material response may also be modelled using strength models such as Steinberg-Guinan [31] and Johnson-Cook [32]. CTH has been successfully applied to the study of granular ceramic materials impacted in a velocity regime similar to that discussed in this paper [28]. The CTH code contrasts with implicit finite element codes in which the main aim is to simulate the response of materials in the low strain rate regime where the response of material is dominated by strength rather than by inertial effects.

A summary of the findings of our earlier study is as follows. Both "full" and "cut down" three-dimensional structural CTH calculations were run in which the stereo lithography (stl) files used to manufacture the samples were imported into CTH. In these simulations the detailed, dimensioned, structure of the whole cellular sample was modelled but in the "cut down" simulations only a 2 cell $\times 2$ cell $\times 6$ cell column of cells was modelled thereby much reducing the computational requirements. As expected, it was found that, since the conditions in the sample were close to $1 \mathrm{D}$, there was little difference in the predictions of the full and cut down simulations. It was found that both sets of 3D simulations gave a reasonable match to all of the features observed in the experiments including the oscillations which were superimposed on the first and second velocity plateaus at the back surface of the solid steel target. In particular, the simulations provided insights into the processes at play in the shocked lattice. As the compression wave reaches a cavity, a release wave followed by a compression wave is generated. The array of cavities in the structure thereby generates stress oscillations which propagate in all directions. As these waves reach the back surface of the target, they cause the velocity oscillations observed in the experiment. In summary, our study gave us confidence in the capability of CTH to predict material response in this regime and gave some important insights into the mechanisms at play. However, because the structure was rather complex it was not ideally suitable as a basis for a systematic study of the effects of cell design on energy absorption. It was felt, therefore, that our proposed study of the effect of cell shape on energy absorption needed to be based on a structure that was much simpler than that used in the earlier study.

In this paper we describe the use of the previously validated CTH models to perform an extended study of a simplified lattice structure. In the study described here, the simulations are confined to a single $6 \mathrm{~mm} \times 6 \mathrm{~mm}$ twodimensional cell allowing the distortions of the structure to be imaged at higher resolution than in the earlier study. The aim is to provide a clearer understanding of the energy absorbing processes occurring in metal-based cellular samples during impact than was provided by the relatively complex structures in the earlier study and, thereby, to support the optimisation of cellular structures for application as energy absorbers.

\section{Modelling set-up}

In the study described here simulations were confined to a single cell. Further significant savings on computer resources were made by adopting a two-dimensional rather than a three-dimensional configuration. Figure 1 shows an idealised two-dimensional configuration, chosen as the basis for the current study. The impactor is directed vertically upwards. The energy absorbing elements (EAE) and the impactor and target are considered to consist of infinitely long bars extending in and out of the paper. The outlines shown in Fig. 1 represent sections through these bars. For example the grey rectangle illustrates the orientation of the "rod" variant (also shown on the extreme right of Fig. 2). The flyer and the target are rectangles of dimensions $6 \mathrm{~mm} \times 18 \mathrm{~mm}$, again effectively extending an infinite distance in and out of the paper. In order to provide an improved understanding of the effects of lattice structure on energy absorption, it was necessary 
Fig. 1 Schematic of the two-dimensional (2D) configuration used a basis for CTH study of energy absorption; simulations were run with a range of structures in the region shown as a grey square

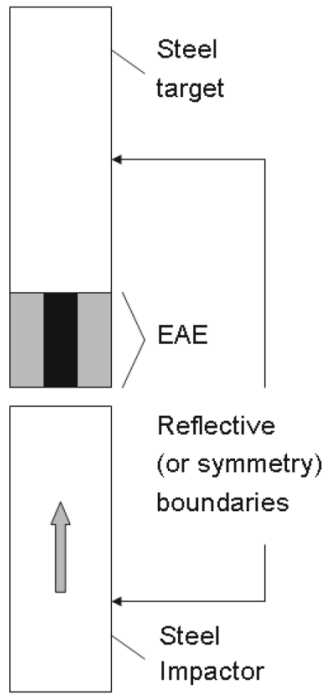

Impactor

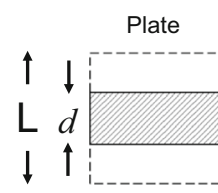

a

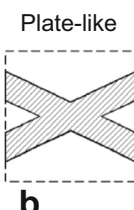

b
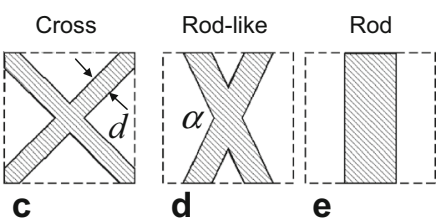

Fig. 2 The five structures assessed using CTH. In all cases the $18 \mathrm{~mm}$ impactor approaches the cell from below. The $18 \mathrm{~mm}$ target block is positioned above, and in contact, with the EAE

to evaluate a systematic range of potential strut orientations. Therefore, simulations of the five alternative EAE structures depicted in Fig. 2 were performed. In all cases the solid occupies $40 \%$ of the total volume of the cell. The flyer impacts from below and the target, or acceptor, is above the EAE. For convenience the EAE on the extreme right (Fig. 2e) is labelled "rod". However, since this is a 2D setup the "rod" element actually consists of a plate extending into the paper. The structure on the extreme left (Fig. 2a) also consists of a plate extending into the paper but, it is rotated by $90^{\circ}$ relative to the structure on the extreme right. The structure in the centre, labelled "cross" consists of two plates at $90^{\circ}$ to each other. The inclusion of the rodlike, cross and platelike structures provides a simple transition from rod to plate structure. Since the shape and mass of the unit cell is held constant the strut thickness, $d$, depends solely on the angle $\alpha$. It can be shown that the porosity, $\phi$, defined as the fraction of the cell occupied by solid material, is given by:

$\phi=d / L$

for options a and e

$\phi=\left[\frac{2 d L}{\sin (\alpha / 2)}-\frac{d^{2}}{2 \cos (\alpha / 2) \sin (\alpha / 2)}\right] \frac{1}{L^{2}}$

Table 1 Structural parameters for the 5 EAE designs

\begin{tabular}{llllll}
\hline & Rod & Rodlike & Cross & Platelike & Plate \\
\hline$\alpha\left(^{\circ}\right)$ & 0 & 130 & 90 & 50 & 180 \\
$d(\mathrm{~mm})$ & 2.4 & 1.24 & 0.96 & 1.24 & 2.4 \\
\hline
\end{tabular}

for options $b$ and $d$

$\phi=\left[d \sqrt{8 L^{2}}-2 d^{2}\right] \frac{1}{L^{2}}$

for option c where $\alpha, d$ and $L$ are as defined in Fig. 2.

It is envisaged that, theoretically, each of the unit cells depicted in Fig. 2 could be repeated horizontally and vertically to provide a macroscopic structure. However, note that, with the exception of the "cross", the macroscopic structure that would be formed by repeating the unit cells studied here would not form practicable, interconnected, material Structural parameters for the five EAE designs are shown in Table 1.

The steel components were modelled using a MieGruneisen equation of state and the Steinberg-Guinan (SG) strength model, the same approach used in the previously reported study. The SG model is implemented as follows. The yield stress, $Y$, is given by:

$$
\begin{aligned}
Y= & \min \left\{Y_{0}\left(1+\beta \varepsilon_{\mathrm{PL}}\right)^{n}, Y_{\max }\right\} \\
& \times\left(1+g \frac{p}{\eta^{1 / 3}}-h(T-300)\right) \\
& \times \exp \left(-0.001 \frac{T}{T_{\text {melt }}(p)-T}\right)
\end{aligned}
$$

where $Y_{0}$ is the yield stress in the reference state, $p$ is the pressure, $\eta$ the density normalized by the reference solid density, and $T$ is the temperature. $\varepsilon_{\mathrm{PL}}$, is the equivalent plastic strain, and $\beta, n, g, h$ are material-dependent parameters. The first term on the right of this equation represents work hardening, and the second term pressure hardening and thermal softening. The final, exponential, term produces a rapid decay in flow stress as the melt temperature is approached. The material parameters used in the current study are shown in Table 2 . They were the same as those which have been used in the earlier investigation apart from the yield strength. The $\mathrm{CTH}$ default value for $Y_{0}(0.34 \mathrm{GPa})$ was used in the current calculations whereas in the previous study an elevated value of $0.64 \mathrm{GPa}$, based on the measured strength of SLM steel was used. The mesh size in the study reported here was $0.02 \mathrm{~mm}$ as compared with $0.05 \mathrm{~mm}$ in the earlier work. In this work, the number of cells through the thickness of each strut varied between 48 and 120 depending on which EAE was being investigated; this compares with $\sim 8$ cells through each strut in the earlier study [29]. 
Table 2 Constants used in the Mie-Gruneisen equation of state and the Steinberg strength model for steel

\begin{tabular}{|c|c|c|}
\hline Density, $\rho_{0}$ & $\mathrm{~g} \mathrm{~cm}^{-3}$ & 7.90 \\
\hline Specific volume, $v_{0}$ & $\mathrm{~cm}^{3} \mathrm{~g}^{-1}$ & 0.1266 \\
\hline Initial temperature, $t_{0}$ & $\mathrm{~K}$ & 298 \\
\hline Bulk sound speed, $C_{0}$ & $\mathrm{~mm} \mu \mathrm{s}^{-1}$ & 4.569 \\
\hline $\begin{array}{l}\text { Slope of shock velocity vs. } \\
\text { particle velocity plot, } S\end{array}$ & & 1.49 \\
\hline Gruneisen gamma, $\Gamma_{0}$ & & 2.17 \\
\hline $\begin{array}{l}\text { Specific heat at constant } \\
\text { volume, } C_{\mathrm{v}}\end{array}$ & $\mathrm{J} \mathrm{g}^{-1} \mathrm{~K}^{-1}$ & 0.446 \\
\hline Initial flow stress, $Y_{0}$ & $\mathrm{GPa}$ & 0.34 \\
\hline Constant in (4), $\beta$ & & 43 \\
\hline Constant in (4), $n$ & & 0.35 \\
\hline Maximum flow stress, $Y_{\max }$ & $\mathrm{GPa}$ & 2.5 \\
\hline Constant in (4), $g$ & $\mathrm{GPa}^{-1}$ & 0.03685 \\
\hline Constant in (4), $h$ & $\mathrm{~K}^{-1}$ & -0.0004545 \\
\hline Melt temperature, $T_{\text {melt }}$ & $\mathrm{K}$ & 2380 \\
\hline Shear modulus, $G$ & GPa & 77.0 \\
\hline Initial temperature & $\mathrm{K}$ & 300 \\
\hline Initial internal energy & $\mathrm{J} \mathrm{g}^{-1}$ & 133 \\
\hline
\end{tabular}

Key objectives of the study to be described are to obtain insights into the mechanisms at play during the interaction between the flyer and the cushioned target and to estimate from simulations which of the chosen variants maximises the irreversible energy generated in the EAE and thereby minimises the energy transferred to the target. Therefore CTH was used to generate time sequences showing materials, plastic flow, internal and kinetic energy, pressure, temperature and flow stress. The total kinetic and total internal energy of the flyer, EAE and target were also output from the code. As mentioned in Sect. 1, the regime of prime interest in this work is the period during which the porous structure (the EAE) compresses due to the transmission of the first plastic wave. However, the transmission of the wave into the solid target is also of great interest because of its potential to evaluate the accuracy of the simulations by monitoring the transmitted wave in future experiments.

\section{Simulations}

Simulations provide a picture of the mechanisms at play during the deformation process following impact. For example Fig. 3 shows a sequence generated by a $0.3 \mathrm{~mm} \mathrm{\mu s}^{-1}$ impact on a rodlike EAE. The left-most frame shows a material plot at time zero. On the distance scale displayed on the extreme left, the solid stainless steel flyer extends from 2 to $20 \mathrm{~mm}$; at time zero it has a velocity of $0.3 \mathrm{~mm} \mathrm{\mu s}^{-1}$ directed ver- tically upwards. The EAE extends from 20 to $26 \mathrm{~mm}$ and the solid steel target extends from 26 to $44 \mathrm{~mm}$. In the rest of the frames, the colours represent velocities with magnitudes indicated by the scale at the extreme right. The times of the frames in microseconds from impact are shown at the top of each frame. From this sequence we judge that full densification of the EAE occurs at $\sim 14 \mu$ s. The estimated trajectories of the waves propagating through the system are shown by the superimposed black lines. The solid black line which extends from the 2.8 to the $14.0 \mu$ s frame shows the estimated trajectory of the front face of the flyer. The dashed lines are a schematic representation of the wavelets generated as the flyer interacts with the EAE. Reference to the velocity scale shows that, by the time full densification of the EAE occurs, the velocity of the flyer has reduced from 0.3 to $\sim 0.24 \mathrm{~mm} \mathrm{\mu s}^{-1}$.

As expected the different EAEs respond differently. Figure 4 shows internal energy, plastic strain and temperature plots for the five EAE variants just after full densification for a simulation at $0.3 \mathrm{~mm} \mathrm{\mu s}^{-1}$. It is clear that the plastic strain and, therefore, internal energy absorbed by the EAE tends to increase as the configuration is varied from plate to rod but that there is little difference between the rodlike and rod variants.

The energies in each computational cell of each of the three regions (flyer, EAE, target) can be summed to give the total energy for a $1 \mathrm{~cm}$ length (measured into the paper) of each region or component as a function of time. As an illustration, Fig. 5 shows plots of internal, kinetic and total (internal plus kinetic) energy for a system with a cross EAE impacted at $0.3 \mathrm{~mm} \mathrm{\mu s}^{-1}$. The kinetic energy in the impactor reduces to a minimum and then rises again. Meanwhile the internal energy exhibits a "double peak" profile which is also seen in the target. The kinetic energy in the target exhibits a single peak over the time frame of the graph. The general shapes of the profiles, which were found to be similar for all of the EAEs, will be discussed further in Sect. 5 .

Figure 6a shows the total internal energy delivered to the different EAEs by a $0.3 \mathrm{~mm} \mu \mathrm{s}^{-1}$ impact. It is seen that the internal energy in all of the EAE initially ramps up and then reaches a plateau. The onset of the plateau corresponds roughly to the time at which the EAE reach full densification. The greatest energy absorption is achieved with the rod and rodlike EAEs and the least energy is absorbed by the plate and platelike EAEs. The cross structure lies between these two. Corresponding results for a $0.9 \mathrm{~mm} \mathrm{\mu s}^{-1}$ impact are shown in Fig. 6b. It can be seen that, again, the energy rises roughly linearly to a plateau. Again the onset of the plateau corresponds to full densification (see also the sequence in Fig. 14). There are two significant differences between the 0.3 and $0.9 \mathrm{~mm} \mathrm{\mu s}^{-1}$ results. First, the internal energies dissipated in the $0.9 \mathrm{~mm} \mathrm{us}^{-1}$ simulation are at least twice the corresponding energies in the $0.3 \mathrm{~mm} \mathrm{\mu s}^{-1}$ simula- 
Fig. 3 A time series showing velocity maps of a simulation of a $0.3 \mathrm{~mm} \mathrm{\mu s}^{-1}$ flyer impacting a rodlike EAE. The time axis is distance-time plot is shown superimposed on the $\mathrm{CTH}$ sequence horizontal. A schematic

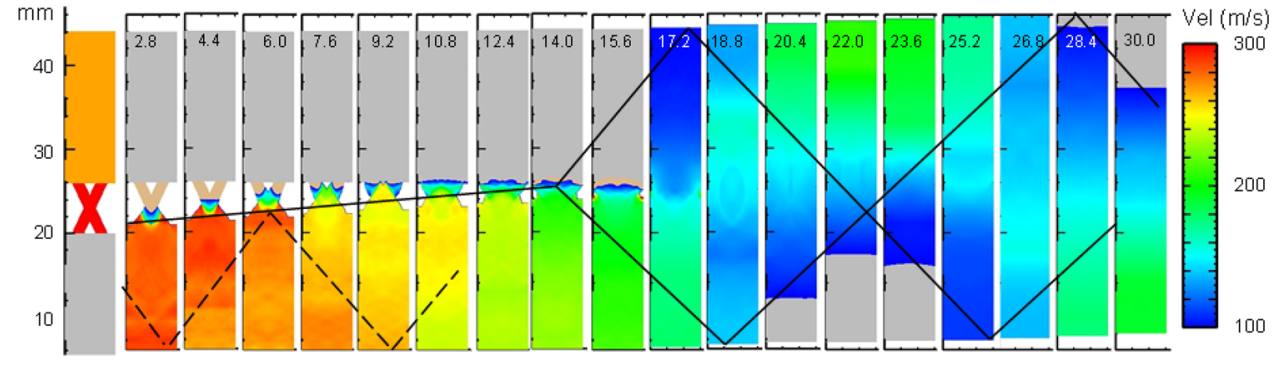

Fig. 4 The effect of cell geometry on plastic strain, internal energy and temperature during a $0.3 \mathrm{~mm} \mathrm{\mu s}^{-1}$ impact. Internal energy is shown at both early and late times. Plastic strain is shown at late times only
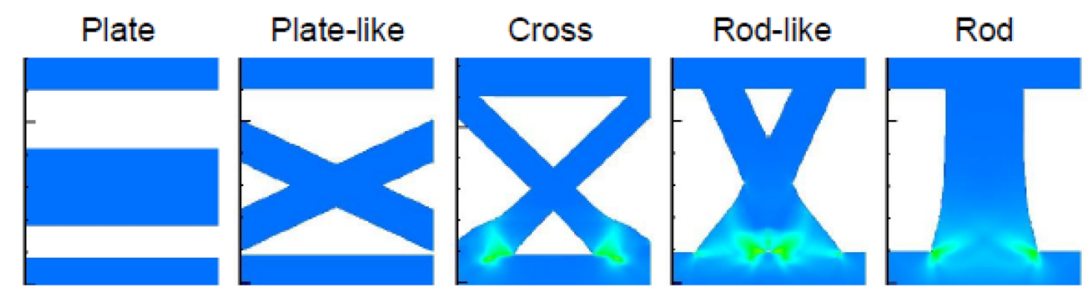

Internal

Energy -

Early Time
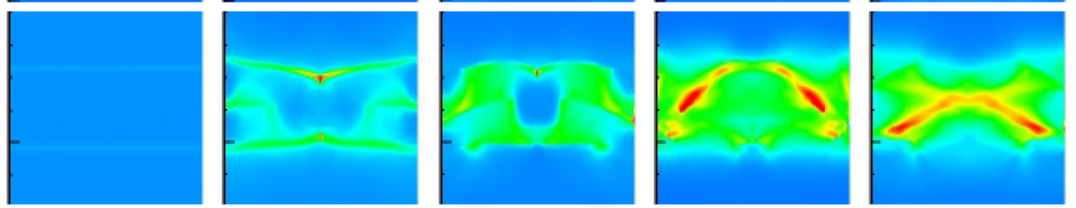

Internal

Energy Late Time
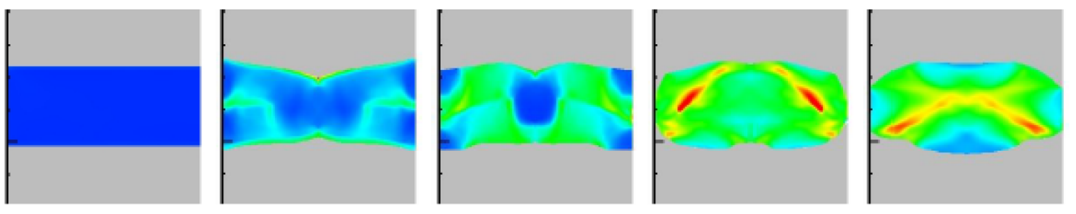

Plastic

Strain -

Late Time
Fig. 5 Computed energies for a system with a cross EAE impacted at $0.3 \mathrm{~mm} \mathrm{\mu s}^{-1}$

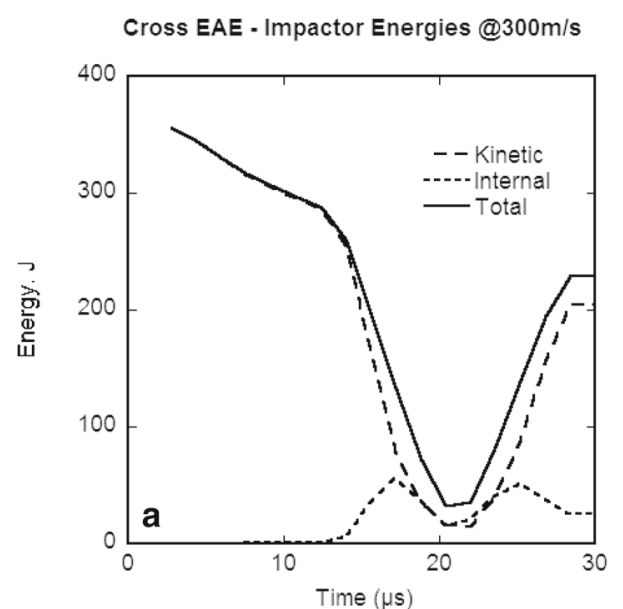

Cross EAE - Target Energies@300 m/s

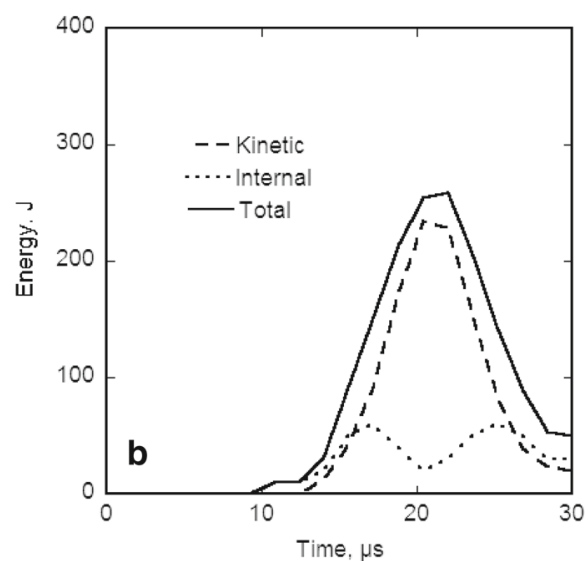

tions. Second, in the higher velocity simulation the energy generated in the cross structure is similar to that generated in the rod and rodlike structures whereas at $0.3 \mathrm{~mm} \mathrm{\mu s}^{-1}$ the cross is intermediate between the rod and plate structures.

One of the objectives of this study was to determine the factors affecting the energy transferred to the target at late time. This is difficult to assess thoroughly using a Eulerian code for two main reasons. First, as explained in the discus- sion, the waves generated by impact will reflect back and forth in the longitudinal direction for a significantly longer time than it was possible to run the simulations in this study. Second, it will be noted later that the model used in the simulations does not allow the components to separate when tensile forces develop between them. It follows that the simulations become invalid before energy exchange between the target and the other components in the system ceases. However, despite this difficulty, we believe a useful guide to the 
Fig. 6 Total energies of a the impactor and $\mathbf{b}$ the target for the five alternative energy absorbing elements
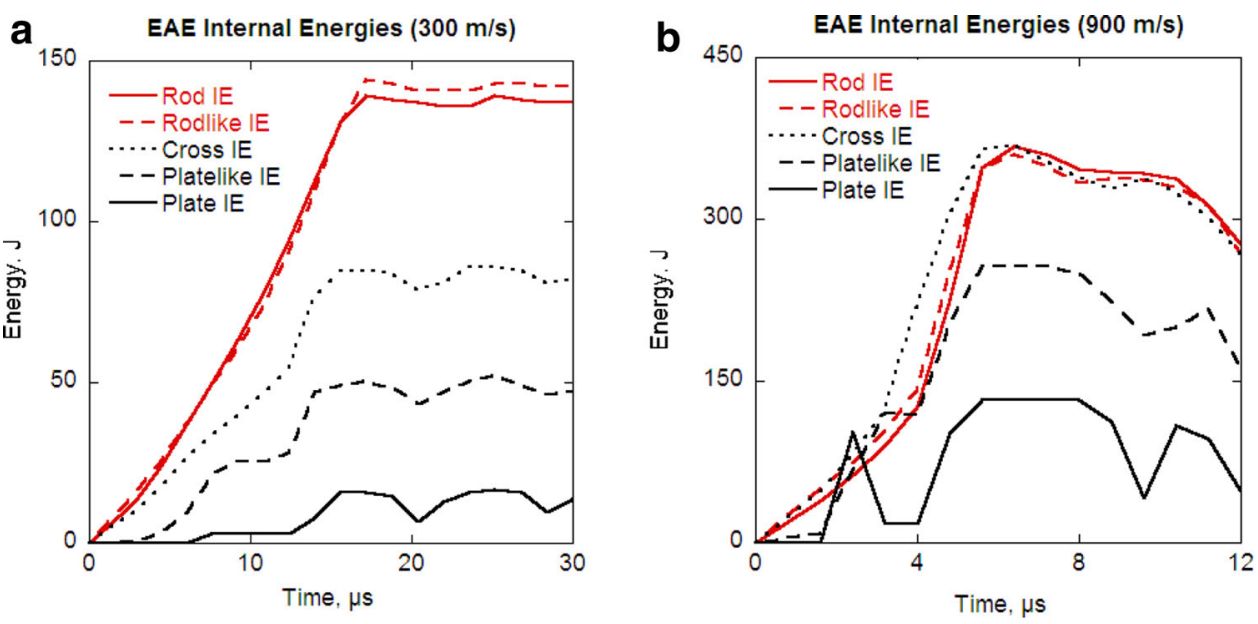

Fig. 7 Total energies (internal + kinetic) generated in the targets. $\mathbf{a}$ and $\mathbf{b}$ shows results for 0.3 and $0.9 \mathrm{~mm} \mathrm{\mu s}^{-1}$ impacts respectively

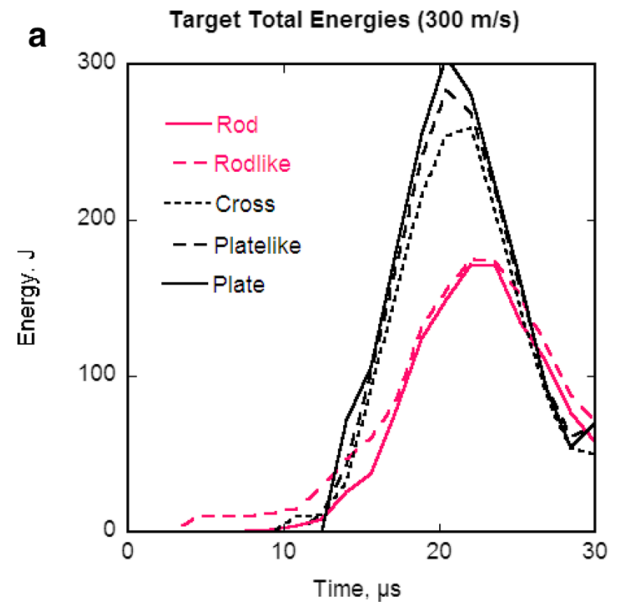

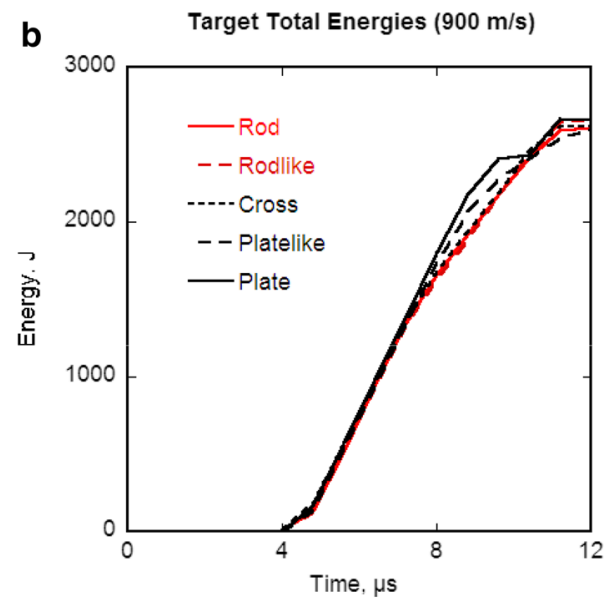

energy transferred to the target can be gained by assessing the energy transferred up to the point at which the forces between the target and the EAE become negative. Figure 7a shows the total (kinetic plus internal) energies in the target following a $0.3 \mathrm{~mm} \mathrm{\mu s}^{-1}$ impact onto a target protected by the five EAEs under investigation. Note that with the rod EAE the energy in the target increases at $\sim 3 \mu$ s after impact. We assume that this occurs as the elastic wave generated by the first impact arrives at the target. By contrast the other EAEs do not transmit a significant elastic wave. In each case the energy rises to a peak and the timing of the peak broadly corresponds to the moment that the release waves reflected from the back face of the flyer and the front face of the target meet. It is clear from Fig. 7a that the peak energy delivered to the target is less for the rod and rodlike EAE than for the plate and platelike EAEs. Figure $7 \mathrm{~b}$ shows the target total energies for a series of simulations of $0.9 \mathrm{~mm} \mathrm{\mu s}^{-1}$ impacts. In these examples the simulations were run as far as the peak energy but no further. Again the position of the peak corresponds to the time at which the stress at the impactor EAE interface is expected to go negative. In this high velocity example, it can be seen that the choice of EAE makes only a very small difference to the energy transferred to the target.

\section{Discussion}

\subsection{Outline}

A series of simulations have been performed with the aim of ranking a series of Energy Absorbing Elements (EAEs) or "cushions" in terms of their effectiveness in minimising the energy transmitted from an impactor with velocities in the range $0.3-0.9 \mathrm{~mm} \mathrm{\mu s}^{-1}$ to an initially stationary target. The results have been presented in the preceding sections. In this section, the aim is to support an understanding of the results by analysing the way the internal energy generated by the impacts is distributed. 


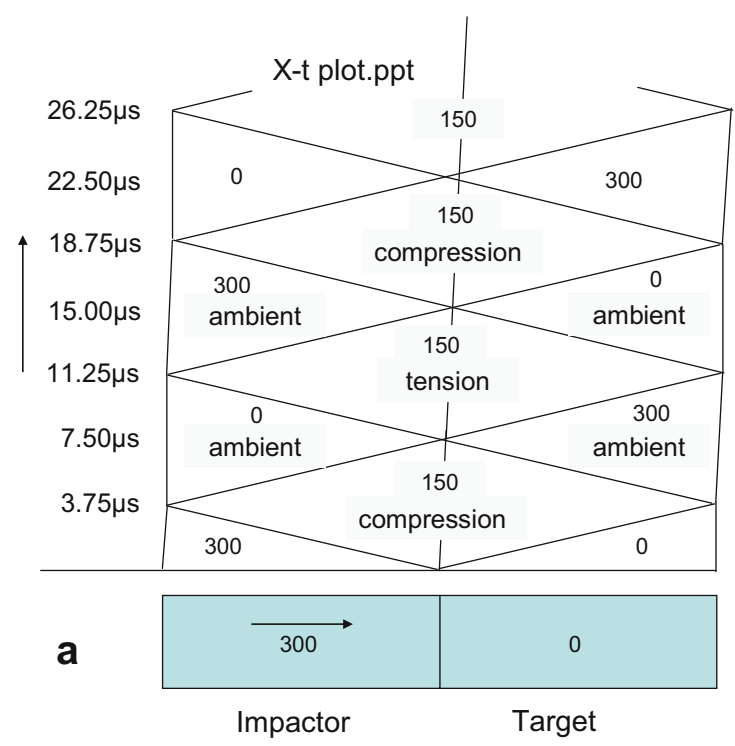

Fig. 8 a Schematic wave patterns following a collision between a $0.3 \mathrm{~mm} \mathrm{\mu s}^{-1}$ solid steel impactor and a solid steel target. The time axis is vertical. The particle velocities (directed to the right) in the different

\subsection{Shock analysis}

Before examining the detailed response of our chosen system (as depicted in Figs. 1,2) it is illustrative to consider the energy distribution in a very simple system from which the EAE has been removed. Figure 8 a shows a schematic distance vs. time plot for a $0.3 \mathrm{~mm} \mathrm{\mu s}^{-1}$ system. For simplicity it has been assumed that energy dissipation is zero and, therefore, that the system remains isentropic. In this situation, all energy transfers are reversible. Therefore the energy is in the form of either kinetic energy or compression energy; effectively the material is treated as elastic. In this situation the wave velocity remains constant (at $4.8 \mathrm{~mm} \mathrm{\mu s}^{-1}$ say) and the particle velocity in between the shocks generated by the impact in the flyer and target is half the impactor velocity. On wave reflection from the free surfaces of the flyer and the target, the surface velocity is exactly twice the in-situ particle velocity. It is also convenient to assume that when the release waves from the free surfaces meet the interface between the flyer and the target, the target and the flyer do not separate. This last assumption, albeit nonphysical, allows a direct comparison with the computer simulations, which were run in a mode which does not allow separation of the components. This aspect of the modelling will be discussed later. The assumptions listed above allow the shock parameters in the flyer and target (e.g., density, pressure, particle velocity and internal energy) to be estimated using basic shock theory.

Knowledge of the particle velocity distribution in the impactor and target allows the kinetic energies (KE) of the impactor and target to be derived. Assuming the density of

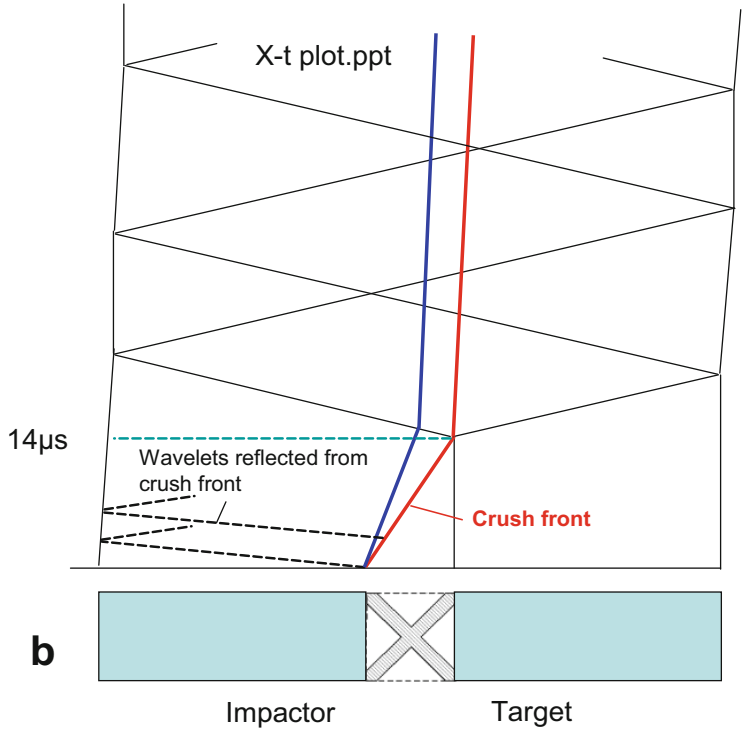

regions are indicated. b Schematic wave patterns following a collision between a $0.3 \mathrm{~mm} \mathrm{\mu s}^{-1}$ solid steel impactor and a target consisting of an energy absorbing cell and a solid steel target

the steel is $7.9 \mathrm{~g} \mathrm{~cm}^{-3}$, and that the flyer measures $0.6 \mathrm{~cm}$ wide $\times 1.8 \mathrm{~cm}$ long and extends $1 \mathrm{~cm}$ into the page, the volume of the flyer is $1.08 \mathrm{~cm}^{3}$ and its mass, $m$, is $8.532 \mathrm{~g}$. The initial kinetic energy of the flyer, $E^{300}$, is given by

$E^{300}=\frac{1}{2} m V^{2}$

where $V$ is the flyer velocity. Table 3 lists the initial kinetic energy of the flyer and the specific initial kinetic energy of the flyer for impact velocities ranging from 0.3 to $0.9 \mathrm{~mm} \mathrm{\mu s}^{-1}$. The average specific energy of the system is calculated by dividing the initial kinetic energy by the total mass of the system.

Knowing the position of the shock as a function of time allows the KE distribution to be deduced. Retaining the $0.3 \mathrm{~mm} \mathrm{\mu s}^{-1}$ "experiment" as an example, the illustration in Fig. 8 a shows that at 0 and $15 \mu$ s the whole impactor (or flyer) has a velocity of $0.3 \mathrm{~mm} \mathrm{us}^{-1}$ and at 7.5 and $22.5 \mu \mathrm{s}$ the whole flyer is stationary. The energy distributions corresponding to Fig. 8a are shown in Fig. 9. It can be seen that the $\mathrm{KE}$ of the flyer oscillates between a maximum of $383 \mathrm{~J}$ at 0 , $15 \mu$ s etc. and a minimum (of zero) at 7.5, $22.5 \mu$ s etc. It can also be seen that (since kinetic energy varies as velocity ${ }^{2}$ ) at $3.75 \mu \mathrm{s}$ (the wave transit time) the kinetic energy of the whole system (flyer plus target) is $192 \mathrm{~J}$ (half the total KE at $0 \mu \mathrm{s}$ ). Therefore at $7.5 \mu \mathrm{s}$ (and $15 \mu \mathrm{s}$, etc.) half the total energy of the system is in kinetic energy and half is in internal energy. Of course, in our simple elastic example, there is no increase of entropy, therefore, at this stage, all of the internal energy is in the form of mechanical compression energy. The 
Table 3 Impactor energies derived using the simple analysis depicted in Figs. 8 and 9

\begin{tabular}{|c|c|c|c|c|c|}
\hline $\begin{array}{l}\text { Flyer velocity } \\
\left(\mathrm{mm} \mu \mathrm{s}^{-1}\right)\end{array}$ & $\begin{array}{l}\text { Mass of flyer } \\
\left(\mathrm{g} \mathrm{cm}^{-1}\right)\end{array}$ & $\begin{array}{l}\text { Mass of EAE } \\
\left(\mathrm{g} \mathrm{cm}^{-1}\right)\end{array}$ & $\begin{array}{l}\text { Initial KE of flyer } \\
\left(\mathrm{J} \mathrm{cm}^{-1}\right)\end{array}$ & $\begin{array}{l}\text { Initial specific KE } \\
\text { of flyer }\left(\mathrm{J} \mathrm{g}^{-1}\right)\end{array}$ & $\begin{array}{l}\text { Average specific } \\
\text { energy of system } \\
\left(\mathrm{J} \mathrm{g}^{-1}\right)\end{array}$ \\
\hline 0.3 & 8.532 & 1.137 & 384 & 45 & 21.1 \\
\hline 0.5 & 8.532 & 1.137 & 1067 & 125 & 58.6 \\
\hline 0.7 & 8.532 & 1.137 & 2090 & 245 & 114.8 \\
\hline 0.9 & 8.532 & 1.137 & 3455 & 405 & 189.9 \\
\hline
\end{tabular}

Fig. 9 Energy distributions in impactor and target predicted by the simple model depicted in Fig. 8a
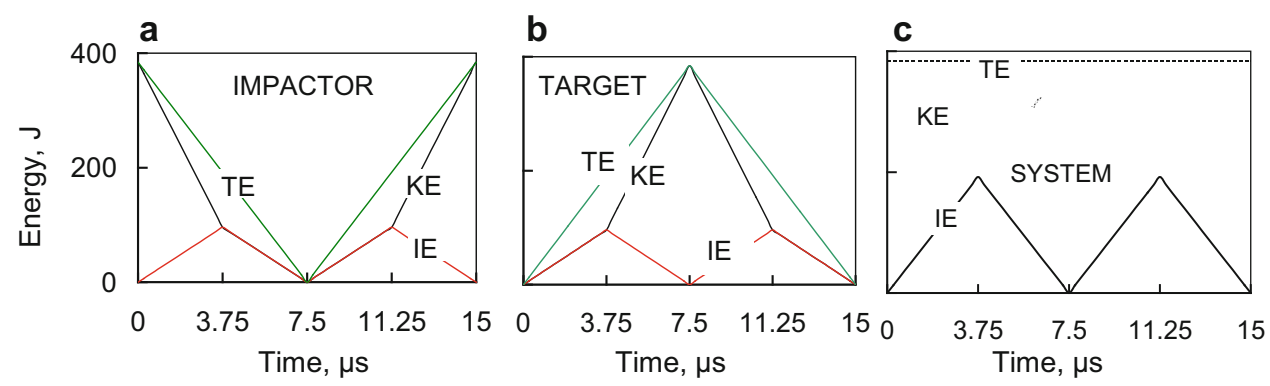

specific internal energy in the initial shock generated by the impact is $11.25 \mathrm{~J} \mathrm{~g}^{-1}$. Note that in both the impactor and the target, the internal energy exhibits a double peak form.

The pressure and specific volume in the shock generated by the impact can be calculated if the Hugoniot of the steel is known. Assuming a linear shock velocity $\left(U_{\mathrm{s}}\right)$ vs. particle velocity $\left(u_{\mathrm{p}}\right)$ relationship and using the constants from Table 2 gives the Hugoniot:

$U_{\mathrm{s}}=4.569+1.49 u_{\mathrm{p}}$

From the laws of conservation for shocks the pressure, $p$, and volume, $v$, behind the initial shock are given by:

$p=\rho_{0} U_{\mathrm{s}} u_{\mathrm{p}}$

and

$v=v_{0}\left(\frac{U_{\mathrm{s}}-u_{\mathrm{p}}}{U_{\mathrm{s}}}\right)$

It can be shown using a pressure versus particle velocity construction that the pressure and specific volume in a shock generated by impacts at a range of velocities are as listed in columns 2 to 4 of Table 4 as are the corresponding normal strains given by:

$\varepsilon_{n}=\ln \left(\frac{v_{0}}{v}\right)$

Note from Fig. 8a that from 7.5 to $15 \mu$ s the interface between the two components is predicted to be in tension. However it is assumed in the illustration that the components will remain in contact. This assumption allows us to compare the predictions of the simple model with the predictions of CTH. Since in reality the components would probably separate rather than go into tension the predictions are not valid after the time the release wave from the front and back surfaces of the system meet (in our example beyond $\sim 22 \mu$ s after impact time). However the results of simulations beyond this time have been presented to permit a more complete comparison between analytical and simulated results. Note that there are models in CTH to treat tensile failure. We plan to investigate the effect of applying these models in future simulations; it is possible that these will affect the late time behaviour of the simulations.

\subsection{Plasticity analysis}

Although the $p, v$ states presented in Table 4 assume that the impactor and target were strengthless, it is useful to separate the internal energy that would have been generated if the material had finite strength into its component parts. Remember that the following analysis applies to a simple metal-on-metal impact with no EAE present.

The red line in the schematic stress vs. strain plot in Fig. 10 depicts the pressure vs. strain response of a material subjected (at a low strain rate) to uniaxial strain. It is assumed that the pressure vs. strain relationship may be simplified as;

$p=K \varepsilon_{n}$

where $K$ is the bulk modulus, and $\varepsilon_{n}$ is given by:

$\varepsilon_{n}=\ln \left(\frac{v_{0}}{v}\right)$ 

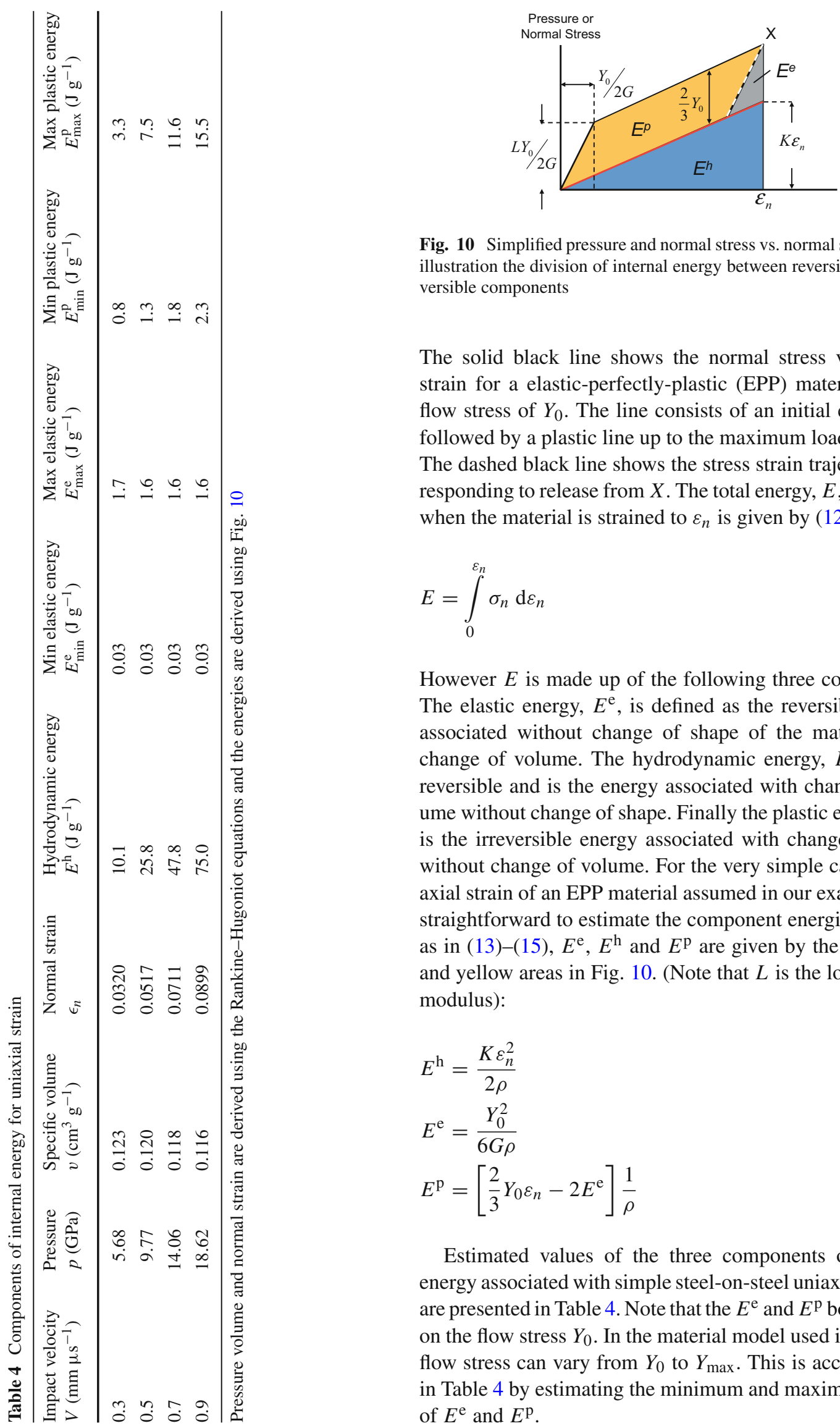

Fig. 10 Simplified pressure and normal stress vs. normal strain curves illustration the division of internal energy between reversible and irreversible components

The solid black line shows the normal stress vs. normal strain for a elastic-perfectly-plastic (EPP) material with a flow stress of $Y_{0}$. The line consists of an initial elastic rise followed by a plastic line up to the maximum load point, $X$. The dashed black line shows the stress strain trajectory corresponding to release from $X$. The total energy, $E$, generated when the material is strained to $\varepsilon_{n}$ is given by (12):

$E=\int_{0}^{\varepsilon_{n}} \sigma_{n} \mathrm{~d} \varepsilon_{n}$

However $E$ is made up of the following three components. The elastic energy, $E^{\mathrm{e}}$, is defined as the reversible energy associated without change of shape of the material with change of volume. The hydrodynamic energy, $E^{\mathrm{h}}$, is also reversible and is the energy associated with change of volume without change of shape. Finally the plastic energy, $E^{\mathrm{p}}$, is the irreversible energy associated with change of shape without change of volume. For the very simple case of uniaxial strain of an EPP material assumed in our example, it is straightforward to estimate the component energies. In fact, as in (13)-(15), $E^{\mathrm{e}}, E^{\mathrm{h}}$ and $E^{\mathrm{p}}$ are given by the grey, blue and yellow areas in Fig. 10. (Note that $L$ is the longitudinal modulus):

$E^{\mathrm{h}}=\frac{K \varepsilon_{n}^{2}}{2 \rho}$

$E^{\mathrm{e}}=\frac{Y_{0}^{2}}{6 G \rho}$

$E^{\mathrm{p}}=\left[\frac{2}{3} Y_{0} \varepsilon_{n}-2 E^{\mathrm{e}}\right] \frac{1}{\rho}$

Estimated values of the three components of internal energy associated with simple steel-on-steel uniaxial loading are presented in Table 4 . Note that the $E^{\mathrm{e}}$ and $E^{\mathrm{p}}$ both depend on the flow stress $Y_{0}$. In the material model used in CTH the flow stress can vary from $Y_{0}$ to $Y_{\max }$. This is accounted for in Table 4 by estimating the minimum and maximum values of $E^{\mathrm{e}}$ and $E^{\mathrm{p}}$. 
Fig. 11 Energy profiles in the configuration depicted in Fig. 3b estimated using the simple model and simulation

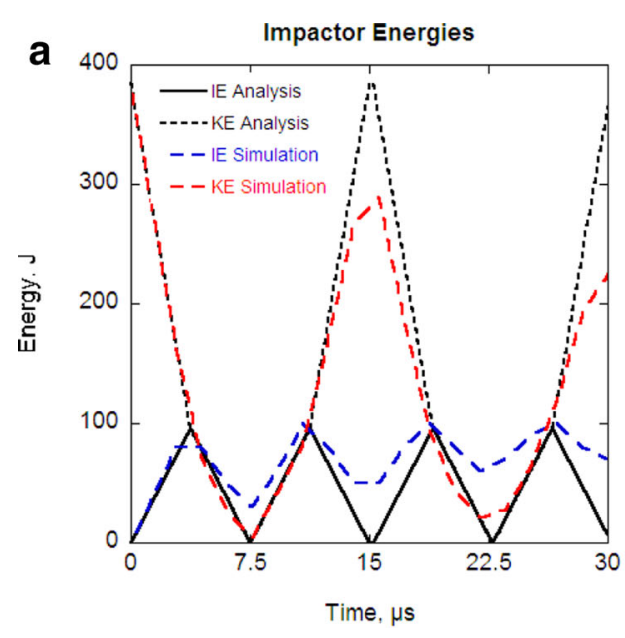

The expected effect of adding an energy absorbing element to the basic configuration shown in Fig. 8a is illustrated schematically in Fig. 8b. The leading edge of the flyer interacts with the leading edge of the EAE, and high pressure wavelets are driven back into the flyer. These wavelets reflect from the back face of the flyer leading to a gradual reduction in its velocity. Simultaneously, we would expect that wavelets driven through the structure of the EAE gradually impart movement and energy to the target.

\subsection{Analysis of simulations}

The wave propagation histories in the simplified model and the energy distributions derived from them furnish a baseline against which to compare the $\mathrm{CTH}$ simulations and provide a qualitative explanation of some of the features observed in the energy vs. time profile generated by the simulations. For example Fig. 11a, b shows computed energies for a system without an EAE for comparison with the idealised configuration depicted in Fig. 8a. It is seen that the periodic time variation in the internal energy profile generated by the simulations corresponds qualitatively to the oscillations between the back face of the flyer and the front face of the target. However, unlike the simple elastic model, the CTH simulation predicts that the amplitude of the oscillatory waves decreases with time. This is because, in the code, the passage of the compression waves converts some of the initial kinetic energy to irreversible energy by shock propagation and plastic flow.

In the example in Fig. 12 the time variation of the computed energies in an $0.3 \mathrm{~mm} \mathrm{\mu s}^{-1}$ impactor following interaction with a rodlike EAE is shown for comparison with the predictions of the simplified configuration in Fig. 8a. It is seen that the shape features obtained from the simulated energy vs. time plots are similar to those implied by the simple analysis. In particular, the comparison provides a clear

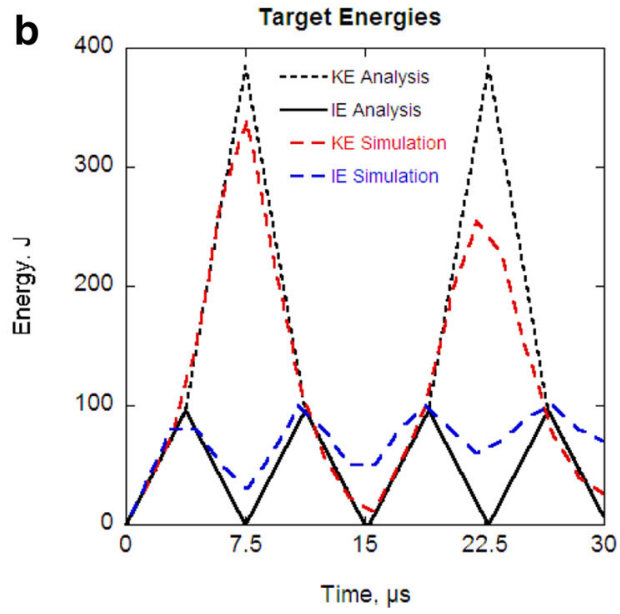

Impactor Energies for Rod EAE (300 m/s)

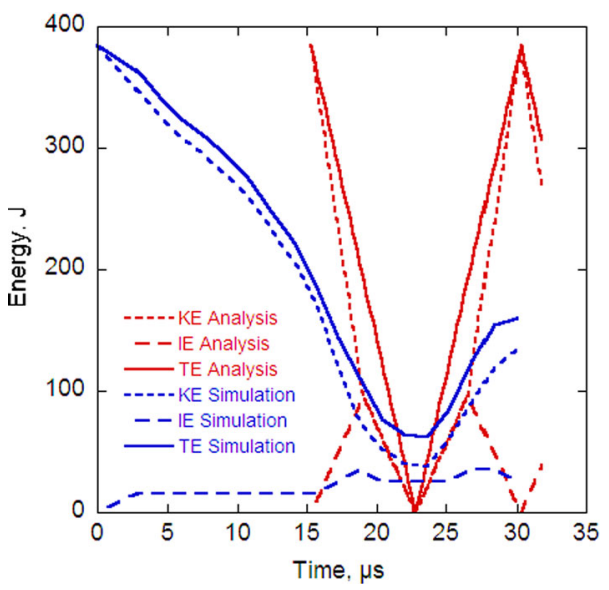

Fig. 12 Internal (IE), kinetic (KE) and total energies (TE) generated in the impactor for $0.3 \mathrm{~mm} \mathrm{\mu} \mathrm{s}^{-1}$ impact onto a "rod" lattice. The red lines were generated using the simple analysis illustrated in Figs. 3 and 4; the blue lines show the values obtained from CTH simulations

qualitative explanation for the double peak in internal energy seen in the simulations.

As with the simple uniaxial strain configuration discussed in the previous section, the total internal energy, $E$, generated in the simulations of the cushioned systems may be regarded as the sum of three components. Remember the compression energy, $E^{\mathrm{c}}$, is the energy associated with changes in volume without change of shape and the elastic energy, $E^{\mathrm{e}}$, is the energy associated with shear distortion prior to yielding. Compressional and elastic energy are reversible and will be available to do work on the system as the forces on the system are relaxed. The plastic energy, $E^{\mathrm{p}}$, is the energy associated with plastic flow following yielding. Plastic energy is not reversible but instead is converted to heat and not, therefore, returned to the system at late time. It follows that maximising the plastic energy generated in the EAE minimises the energy reflected back into the impactor and transmitted into the target. 


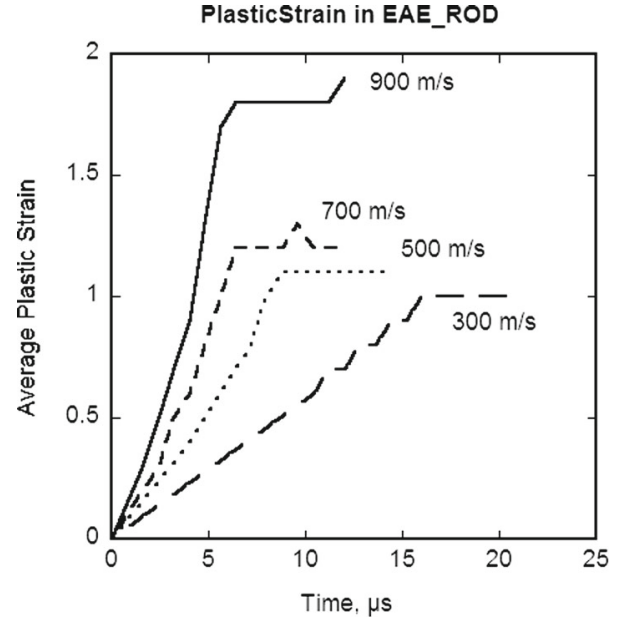

Fig. 13 Plot of average plastic strain in EAE vs. time

Ideally, the plastic energy (or plastic work) generated in each of our EAE variants during densification would be output from the CTH code. Although CTH does not appear to have the capability to output plastic energy it can provide plastic strain. For example Fig. 13 shows the plastic strain averaged over all the cells in rod EAEs generated during loading by projectiles with velocities ranging from 0.3 to $0.9 \mathrm{~mm} \mathrm{\mu s}^{-1}$. In each case there is a roughly linear increase in average plastic strain followed by a plateau. The onset of the plateau corresponds to full densification of the EAE. The simulations clearly show that, as impact velocity increases, the amount of plastic flow corresponding to full densification increases. Since the stress at which this flow takes place would be expected to increase with pressure, the increase in plastic work with velocity would be even more marked than the trend shown in Fig. 13. An alternative approach is illustrated by the data in Table 5 which lists the specific internal energies generated in the EAEs at 0.3 and $0.9 \mathrm{~mm} \mathrm{\mu s}^{-1}$. Also listed in Table 5 are the elastic and compression energies estimated for shock waves generated by steel-on-steel impact without an EAE present. We assume that these latter values represent upper limits on the elastic and compression energy generated in an EAE. It can be seen that, at both impact velocities and for all EAE variants, the elastic component is small compared with plastic energy. For the plate configuration the estimated compression energy is an appreciable fraction of the total internal energy which is not surprising since the plate configuration is geometrically similar to a simple steel-on-steel impact. However it is clear that in the other EAE configurations the total internal energy is dominated by the plastic component of the internal energy. It follows that, in most of the simulations run in support of this investigation, the total internal energy may be taken as an approximate measure of the plastic energy. This contention is supported by the observation in Fig. 4 that the patterns showing the distribution of plastic strain appear very similar to the corresponding internal energy distributions. A possible exception to this trend is the plate EAE where, as might be intuitively expected, most of the internal energy is in the form of hydrodynamic compression energy.

\subsection{Conclusions from energy analysis}

It is clear, and intuitively obvious, that the densification of the rod and rodlike EAEs requires more plastic flow than the platelike and plate options. Consequently, the former absorb more irreversible energy and so transmit less energy to the target.

The amount of plastic flow associated with full densification also depends on the impact velocity. As impact velocity increases the energy analysis indicates that the amount of plastic flow and therefore the irreversible energy generated increases. Additional simulations were run to explore this phenomenon.

The sequence in Fig. 14 maps internal energy density for a rodlike cell impacted at $0.9 \mathrm{~mm} \mu \mathrm{s}^{-1}$. Full densification of the cell occurs at $\sim 4.8 \mu \mathrm{s}$, after which time there is very little change in the internal energy distribution in the vicinity of the EAE. Note that intense flow occurs at the edges of the cell as mushrooming of the angled plates produces jets which eventually impinge on the end-face of the target. Note that the small mesh size $(0.02 \mathrm{~mm})$ employed in this study has allowed the fine detail of the jets and their interaction with the cell walls to be resolved. The highest specific energies seen in the graphics are generated as a result of this jet impact. It can be seen that, following impact, plastic waves propagate into the flyer and the target.

Comparing Fig. 6a with Fig. 6b shows that increasing the impact velocity increases the energy absorption in the EAE and the relative amplitudes of the energy profiles in the target, shown in Fig. 7, are consistent with the amplitudes of the internal energy profiles in the corresponding

Table 5 CTH simulations: peak volume average specific internal energies generated in the EAE $\left(\mathrm{J} \mathrm{g}^{-1}\right)$

\begin{tabular}{lccccccc}
\hline $\begin{array}{l}\text { Impact velocity } \\
\left(\mathrm{mm} \mu \mathrm{s}^{-1}\right)\end{array}$ & Plate $\left(\mathrm{J} \mathrm{g}^{-1}\right)$ & Platelike $\left(\mathrm{J} \mathrm{g}^{-1}\right)$ & Cross $\left(\mathrm{J} \mathrm{g}^{-1}\right)$ & Rodlike $\left(\mathrm{J} \mathrm{g}^{-1}\right)$ & Rod $\left(\mathrm{J} \mathrm{g}^{-1}\right)$ & $\begin{array}{l}\text { Elastic energy } \\
\left(\mathrm{J} \mathrm{g}^{-1}\right)\end{array}$ & $\begin{array}{l}\text { Compression } \\
\text { energy }\left(\mathrm{J} \mathrm{g}^{-1}\right)\end{array}$ \\
\hline 0.3 & 15 & 40 & 74 & 122 & 126 & $<1.7$ & 10 \\
0.9 & 117 & 225 & 324 & 316 & 323 & $<1.7$ & 75 \\
\hline
\end{tabular}


Fig. 14 A CTH sequence showing internal energy generation in a rodlike structure impacted (from below) at

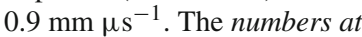
the top are time in microseconds

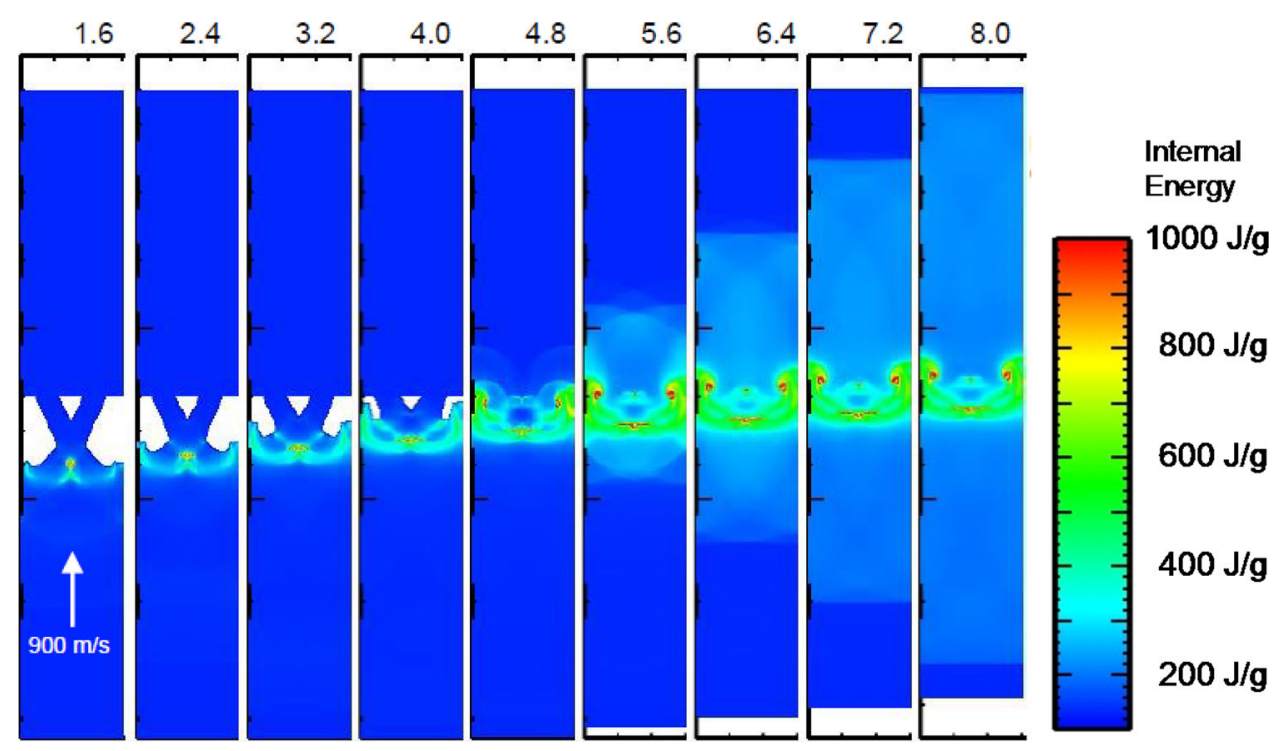

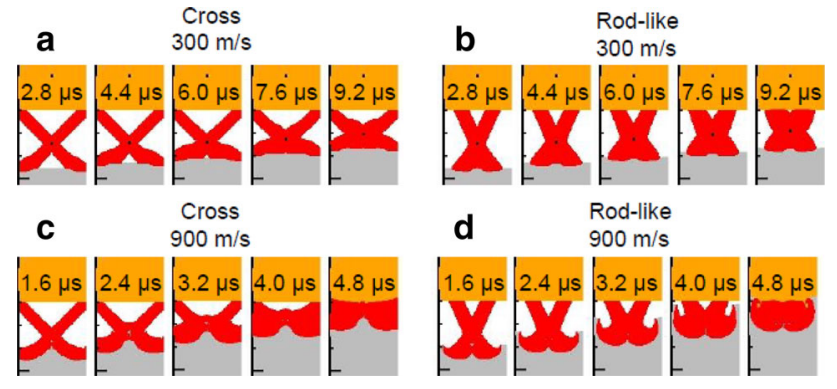

Fig. 15 The top row shows cross vs. rodlike structures at $0.3 \mathrm{~mm} \mu \mathrm{s}^{-1}$ and the bottom row shows a similar comparison at $0.9 \mathrm{~mm} \mathrm{\mu s}^{-1}$

EAEs. Of particular interest here is the observation that, at

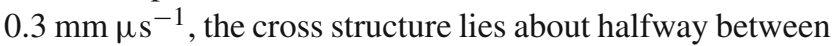
the platelike and rodlike structures, but at $0.9 \mathrm{~mm} \mathrm{us}^{-1}$ there is little difference between the cross and the rod and rodlike structures. The differing effectiveness of the cross structure as the impact velocity changes may be explained by comparing the deformation mechanisms of the cross and rodlike structures at 0.3 and $0.9 \mathrm{~mm} \mathrm{\mu s}^{-1}$. The simulated response of the cross and rodlike structures at 0.3 and $0.9 \mathrm{~mm} \mathrm{\mu s}^{-1}$ are shown for comparison in the material plots in Fig. 15a-d. The frames in Fig. 15a shows that, according to the simulations, at $0.3 \mathrm{~mm} \mathrm{\mu s}^{-1}$ the cross structure closes by axial movement of the parts of the cell driven by the impactor. By contrast, as shown in Fig. $15 \mathrm{~b}$, at $0.3 \mathrm{~mm} \mathrm{\mu} \mathrm{s}^{-1}$ the rodlike cell closes predominantly by lateral flow, a process accompanied by significantly more plastic deformation than with the cross variant. Therefore, at $0.3 \mathrm{~mm} \mathrm{us}^{-1}$, more energy is absorbed by the rodlike than by the cross variant. At $0.9 \mathrm{~mm} \mathrm{\mu s}^{-1}$ different mechanisms come into play. It is seen from Fig. $15 \mathrm{c}, \mathrm{d}$ that both the cross and the rodlike variants deform by a concentration of lateral flow in the region of the cell adjacent to the impactor. This produces a mushroom feature which occu- pies the whole width of the cell and intense lateral flow and jetting predominate. Plots showing plastic strain in cross and rodlike EAEs impacted at $0.9 \mathrm{~mm} \mathrm{\mu s}^{-1}$ are shown in Fig. 16. As indicated, in the cross structure concentrations of flow develop between the impactor and the diagonal struts and between the diagonal struts themselves. Particularly prominent is a mushroom feature located near the original centre of the cross, which we believe is formed by penetration of the cross by a jet of impactor material. These concentrations of deformation, or microkinetic flows, which are not observed when the cross variant is impacted at $0.3 \mathrm{~mm} \mathrm{\mu s}^{-1}$ are the probable reason for the much-increased energy absorption as velocity increases. In summary the similarity of the mechanisms in the two EAEs results in the energy generated in the EAEs by $0.9 \mathrm{~mm} \mathrm{us}^{-1}$ impacts being similar. The change in deformation behaviour at higher velocities to one dominated by intense plastic flow parallels that observed by Nesterenko in studies of granular materials [33]. Nesterenko proposed that the compression of granular porous material at high rates consisted of distortions of the grains themselves combined with smaller scale deformations resulting in features such as jets and localised melting at grain boundaries. Separating the collapse process into two phases enabled the concept of microkinetic energy to be introduced, defined as the energy dissipated via intense plastic flow during pore collapse. In our simulations, it appears that the occurrence of the plastic flow significantly increases the energy absorbing capability of a particular structure, representing the microkinetic energy component of the total dissipated energy.

Figure 17 shows the results of loading a rod structure at velocities increasing from 0.3 to $0.9 \mathrm{~mm} \mathrm{\mu s}^{-1}$. The simulations clearly show that as the impact velocity is increased beyond $0.5 \mathrm{~mm} \mathrm{us}^{-1}$ there is a transition from a mechanism dominated by roughly uniform lateral flow to one domi- 
Fig. 16 Plastic strain plots for cross and rodlike structures

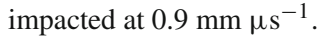
Typical strain concentrations are indicated by arrows
Fig. 17 Internal energy plots for the rod structure. The four columns show the results of simulations at $0.3,0.5,0.7$ and $0.9 \mathrm{~mm} \mathrm{\mu s}^{-1}$, all plotted using the internal energy scale shown. It is evident that the total internal energy in the EAE at the fully densified stage increases with impact velocity
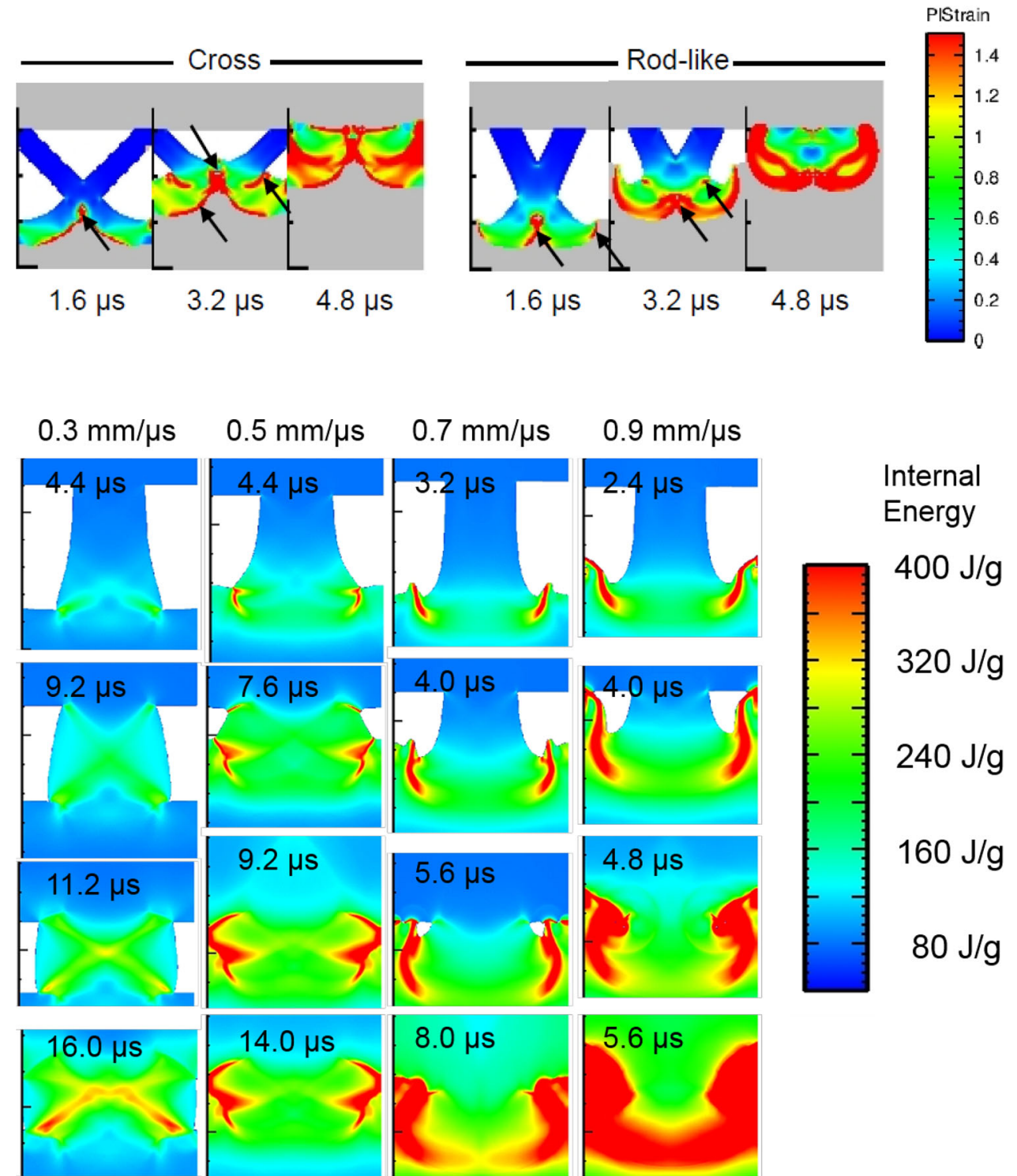

nated by jetting. The results depicted in Figs. 15 and 16 may be compared with Borg and Vogler's CTH simulations of granular ceramic materials impacted at velocities

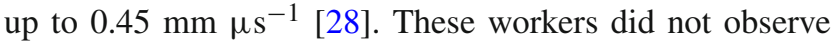
microjetting in their simulations. Our work is consistent with their findings in that microjetting was observed at impact velocities of 0.7 and $0.9 \mathrm{~mm} \mathrm{\mu s}^{-1}$ but not at 0.3 and $0.5 \mathrm{~mm} \mathrm{\mu s}^{-1}$.

Note that intense flow concentrations have been observed both in the microjets and in the material with which the jets interact. Typically, in the simulations depicted in Figs. 14, 15,16 and 17, flow concentrations have length scales extending down to the $100 \mu \mathrm{m}$ regime. Although we have not yet made a systematic study of the effect of mesh size on jet formation and interaction, we have observed that in relatively coarse mesh simulations the jets appear much less well defined with a tendency to appear shorter and broader. There- fore we assume that coarsening the meshes from their current value of $20 \mu \mathrm{m}$ would result in significant loss of the detail associated with the microkinetic flows.

Although the simulations have clearly demonstrated that increasing the impact velocity increases the energy absorbed by the EAE, they have also shown that at the highest velocities, the existence of a "single cell" cushion makes negligible difference to the energy transmitted to the target (see Fig. 7b). The reason for this is that the irreversible energy absorbed by the EAE is only a small fraction of the total energy delivered to the system by the impactor. From Table 3 the kinetic energies of the impactor are 384 and $3455 \mathrm{~J}$ at 0.3 and $0.9 \mathrm{~mm} \mathrm{us}^{-1}$ respectively. Multiplying the specific energies listed in Table 4 by the EAE mass $(1.137 \mathrm{~g})$ gives the maximum plastic energy generated in the EAEs as $143 \mathrm{~J}$ and $367 \mathrm{~J}$ at 0.3 and $0.9 \mathrm{~mm} \mathrm{us}^{-1}$ impacts respectively. The fraction of the input energy dissipated in the EAE is, therefore 37 
and $10 \%$ respectively. We note that the effectiveness of an energy absorbing cushion of the type investigated here could be increased by increasing its axial length. This could be achieved by stacking several EAE cells together along the impact direction.

\section{Summary and conclusions}

Porous materials have potential as energy absorbers in shock and impact scenarios. Computer simulation is needed to assess the relative merits of different cellular structures and materials in different loading environments. We have noted that the most useful comparisons between simulation and experiment are those in which nominally one-dimensional, or uniaxial strain, conditions persist, at least until maximum (or full) densification of the porous material is reached. Adopting this configuration has allowed very fine mesh simulation (48-120 meshes through each strut). We note that $1 \mathrm{D}$ conditions are most readily achieved in high density (low porosity) high velocity systems. For this reason we chose to study the response of materials of $40 \%$ solid density in the velocity range 0.3 to $0.9 \mathrm{~mm} \mathrm{\mu s}^{-1}$.

A series of two-dimensional cellular structures designated "Energy Absorbing Elements" (EAEs) have been studied. The main variable was the angle of the flat plates which form the structure. As the angle changed the thickness of the plates was modified to maintain the average density of the structure at $40 \%$ of solid.

The Sandia National Laboratories code, CTH, was used to simulate a system in which an $18 \mathrm{~mm}$ steel flyer impacts a target consisting of a $6 \mathrm{~mm}$ EAE in contact with an $18 \mathrm{~mm}$ steel target. The very fine mesh scheme $(0.02 \mathrm{~mm})$ employed in the code allowed the mechanisms at play during the compaction of the different EAEs to be assessed in various ways. For example, time sequences showing the distribution of particle velocity, pressure, internal energy and plastic strain were plotted. Simulations run at the relatively low velocity of $0.3 \mathrm{~mm} \mathrm{\mu s}^{-1}$ showed that all EAE variants were compressed to solid density in about the same time. However, significantly more plastic flow and internal energy was generated by rod and rodlike EAEs than in the plate and platelike EAEs. It was shown that at the low impact velocity, for most cell types the cells densify by simple broadening of the elements of the EAE. By contrast, as impact velocity is increased to $0.5 \mathrm{~mm} \mathrm{us}^{-1}$ and beyond, jetting processes play an increasingly important role. In effect, jetting increases the amount of plastic flow associated with the full densification of the porous material. The increased plastic flow is accompanied by an increase in the irreversible energy absorbed during the densification process. Quantitative comparison of the effectiveness of the different EAEs can be provided by using $\mathrm{CTH}$ to calculate the energy distribution in the sys- tem. For example the pictorial evidence indicated that more energy was generated in the rod and rodlike EAE than in the platelike versions, implying that the former variant would provide a more effective cushion than the latter. This conclusion was supported by CTH calculations in which the energies generated in each cell of each of the three components of the system were summed. For example, simulations at $0.3 \mathrm{~mm} \mathrm{\mu s}^{-1}$ showed that, relative to a platelike EAE, the rodlike EAE led to a lower gain in total energy of the target.

A very simple analysis of a configuration in which an $18 \mathrm{~mm}$ steel flyer impacts an $18 \mathrm{~mm}$ steel target with no cushion included has been presented. The model provides a prediction of the time variation of the kinetic and internal energies within the flyer and the target, thereby forming a baseline against which to compare simulations of the cushioned systems. The approach provides a qualitative explanation of key features of the simulated energy distribution in the cushioned system and allowed us to estimate the fraction of internal energy dissipated as irreversible energy in the cushioned systems. It was concluded that, apart from the plate variant, the great majority of the internal energy generated in the EAE is irreversible.

By applying the principles presented above when considering the merits of alternative energy absorbing structures, it should be possible to assess which is most suited to a specific impact regime. In conjunction with the close control over cellular material design which is possible using SLM, this approach offers the opportunity to manufacture and test cellular configurations which maximise generation of microkinetic energy.

An important future aim will be to extend the single cell study to multiple cells. In particular simulations will be performed to determine whether the energy absorption scales as cells are stacked in the direction of application of the load.

Further future investigations will consider how to verify the simulations described here against experimental data. One method of accessing this data is dynamic x-ray imaging of the impacted lattice, which will supply line of sight mass at different positions and times. See, for example, a recent study of lattice compaction by Eakins and Chapman [34]. Corresponding deformation profiles can then be extracted from 3D simulations for comparison with experiment.

Acknowledgements AWE and Imperial College London are acknowledged for their continuing support of the Institute of Shock Physics.

Open Access This article is distributed under the terms of the Creative Commons Attribution 4.0 International License (http://creativecomm ons.org/licenses/by/4.0/), which permits unrestricted use, distribution, and reproduction in any medium, provided you give appropriate credit to the original author(s) and the source, provide a link to the Creative Commons license, and indicate if changes were made. 


\section{References}

1. Gibson, L.J., Ashby, M.F.: Cellular Solids - Structure and Properties, 2nd edn. Cambridge University Press (1997)

2. Lu, G., Yu, T.: Energy Absorption of Structures and Materials. Woodhead Publishing Ltd (2003)

3. Han, F., Zhu, Z., Gao, J.: Compressive Deformation and Energy Absorbing Characteristic of Foamed Aluminium. Metall. Mater. Trans. A 29, 2497-2502 (1998). doi:10.1007/s11661-998-0221-Z

4. Hanssen, A.G., Enstock, L., Langseth, M.: Close Range Blast Loading of Aluminium Foam Panels. Int. J. Impact Eng. 27, 593-618 (2002). doi:10.1016/s0734-743x(01)00155-5

5. Nieh, T.G., Higashi, K., Wadsworth, J.: Effect of Cell Morphology on the Compressive Properties of Open-Cell Aluminium Foams. Mat. Sci. Eng. A 283, 105-110 (2000). doi:10.1016/ s0921-5093(00)00623-7

6. Avalle, M., Belingardi, G., Montanini, R.: Characterization of Polymeric Structural Foams Under Compressive Impact Loading by Means of Energy-Absorption Diagram. Int. J. Impact Eng. 25, 455472 (2001). doi:10.1016/s0734x(00)00060-9

7. Li, Q.M., Mines, R.A.W., Birch, R.S.: The Crush Behaviour of Rohacell-51WF Structural Foam. Int. J. Solids Struct. 37, 63216341 (2000). doi:10.1016/s0020-7683(99)00277-2

8. Sherwood, J.A., Frost, C.C.: Constitutive Modelling and Simulation of Energy Absorbing Polyurethan Foam Under Impact Loading. Polym. Eng. Sci. 32, 1138-1146 (1992). doi:10.1002/ pen.760321611

9. Mills, N.J., Zhu, H.X.: The High Strain Compression of Closed Cell Polymer Foams. J. Mech. Phys. Solids 47, 669-695 (1999). doi:10.1016/s0022-5096(98)00007-6

10. Carruthers, J.J., Kettle, A.P., Robinson, A.M.: Energy Absorption Capability and Crashworthiness of Composite Material Structures: A Review. Appl. Mech. Rev. 51, 635-649 (1998). doi:10.1115/1. 3100758

11. Farley, G.L.: Energy Absorption of Composite Materials. J. Compos. Mater. 17, 267-279 (1983). doi:10.1177/ 002199838301700305

12. Morton, J., Godwin, E.W.: Impact Response of Tough Carbon Fibre Composites. Comp. Struct. 13, 1-19 (1989). doi:10.1016/ 0263-8223(89)90069-x

13. Goldsmith, W., Sackman, J.L.: An Experimental Study of Energy Absorption in Impact on Sandwich Plates. Int. J. Impact Eng. 12, 241-262 (1992). doi:10.1016/0734x(92)90447-2

14. Crupi, V., Epasto, G., Guglielmino, E.: Collapse Modes in Aluminium Honeycomb Sandwich Panels Under Bending and Impact Loading. Int. J. Impact Eng 43, 6-15 (2012). doi:10.1016/j. ijimpeng.2011.12.002

15. Papka, S.D., Kyriakides, S.: In-plane Compressive Response and Crushing of Honeycomb. J. Mech. Phys. Solids 42, 1499-1532 (1999). doi:10.1016/0022-5096(94)90085-x

16. Reid, S.R., Peng, C.: Dynamic Uniaxial Crushing of Wood. Int. J. Impact Eng. 19, 531-570 (1997). doi:10.1016/ s0734-743x(97)00016-x

17. Tan, P.J., Reid, S.R., Harrigan, J.J., Zou, Z., Li, S.: Dynamic Compressive Strength Properties of Aluminium Foams. Part 1 Experimental Data and Observations. J. Mech. Phys. Solids 53, 2174-2205 (2005). doi:10.1016/j.jmps.2005.05.007

18. Xue, Z., Hutchinson, J.W.: Crush Dynamics of Square Honeycomb Sandwich Cores. Int. J. Numer. Meth. Eng. 65, 2221-2245 (2006). doi:10.1002/nme.15351
19. Wu, E., Jiang, W.S.: Axial Crush of Metallic Honeycombs. Int. J. Impact Eng. 19, 439-456 (1997). doi:10.1016/ s0734-743x(97)00004-3

20. Liu, Y.D., Yu, J.L., Zheng, Z.J., Li, J.R.: A Numerical Study on the Rate Sensitivity of Cellular Materials. Int. J. Solids Struct. 46, 3988-3998 (2009). doi:10.1016/j.ijsolstr.2009.07.024

21. Deshpande, V.S., Fleck, N.A., Ashby, M.F.: Effective Properties of the Octet Truss Lattice Material. J. Mech. Phys. Solids 49, 17471769 (2001). doi:10.1016/s0022-5096(01)00010-2

22. Wang, J., Evans, A.G., Dharmasena, K., Wadley, H.N.G.: On the Performance of Truss Panels with Kagome Cores. Int. J. Solids Struct. 40, 6981-6988 (2003). doi:10.1016/ S0020-7683(03)00349-4

23. Yungwirth, C.J., Radford, D.D., Aronson, M., Wadley, H.N.G.: Experiment Assessment of the Ballistic Response of Composite Pyramidal Lattice Truss Structures. Composites B: Eng. 39, 556569 (2008). doi:10.1016/j.compositesb.2007.02.029

24. Wadley, H.N.G.: Multifunctional Periodic Cellular Metals. Phil. Trans. R. Soc. A 364, 31-68 (2011). doi:10.1098/rsta.2005.1697

25. Lefebvre, L.P., Banhart, J., Dunand, D.C.: Porous Metals and Metallic Foams: Current Status and Recent Developments' Adv. Eng. Mat. 10, 775-787 (2008). doi:10.1002/adem.200800241

26. Yan, C., Hao, L., Hussein, A., Young, P., Raymont, D.: Advanced Lightweight 316L Stainless Steel Cellular Lattice Structures Fabricated via Selective Laser Melting. Materials and Design 55, 533-541 (2014). doi:10.1016/j.matdes.2013.10.027

27. Mines, R.A.W., Tsopanos, S., Shen, Y., Hasan, R., McKown, S.T.: Drop Weight Impact Behaviour of Sandwich Panels with Metallic Micro Lattice Cores. Int. J. Impact Eng. 60, 120-132 (2013). doi:10.1016/j.ijimpeng.2013.04.007

28. Borg, J.P., Vogler, T.J.: Mesoscale Calculations of the Dynamic Behaviour of a Granular Ceramic. Int. J. Solids Struct. 45, 16761696 (2008). doi:10.1016/j.ijsolstr.2007.10.027

29. Winter, R.E., Cotton, M., Harris, E.J., Chapman, D.J., Eakins, D.E., McShane, G.: Plate-impact Loading of Cellular Structures Formed by Selective Laser Melting. Model. Simul. Mater. Sc. 22, 025021 (2014). doi:10.1088/0965-0393/22/2/025021

30. McGlaum, J.M., Thompson, S.L., Elrick, M.G.: CTH: A threedimensional shock wave physics code' Int. J. Impact Eng. 10, 351360 (1990). doi:10.1016/0734-743X(90)90071-3

31. Steinberg, D.J., Cochran, S.G., Guinan, M.W.: A Constitutive model for metals applicable at high strain rates. J Appl. Phys. 51, 1498-1504 (1980). doi:10.1063/1.327799

32. [2.2] Johnson, G.R., Cook, W.H.: A constitutive model and data for metals subjected to large strains, high strain rates and high temperatures. Proc. 7th Int. Symp. Ballistics (The Hague, Netherlands), 541-547 (1983)

33. Nesterenko, V.F., Lazaridi, A.N.: Regimes of shock-wave compaction of granular materials. In: Holzapfel, W.B., Johnson, P.G. (eds.) High Pressure Science and Technology: Proceedings of XII AIRAPT and XXVII EMPRG International Conference, pp. 835837. Gordon \& Breach, New York (1990)

34. Eakins, D.E., Chapman, D.J.: X-Ray Imaging of Subsurface Dynamics in High Z Materials at the Diamond Light Source. Rev. Sci. Instrum. 85, 123708 (2014). doi:10.1063/1.4904275 